\title{
Isotopic Analysis of Deuterated Water via Single- and Double-pulse Laser-induced Breakdown Spectroscopy
}

\author{
M. Burger, ${ }^{1,2, \text { a) }}$ P. J. Skrodzki, ${ }^{1,2}$ L. A. Finney, ${ }^{1,2}$ J. Hermann, ${ }^{3}$ J. Nees, ${ }^{2}$ and I. Jovanovic ${ }^{1,2}$ \\ 1) Department of Nuclear Engineering and Radiological Sciences, University of Michigan, \\ Ann Arbor, MI 48109, USA \\ ${ }^{2)}$ Center for Ultrafast Optical Science, University of Michigan, \\ Ann Arbor, MI 48109, USA \\ 3) Aix-Marseille University, CNRS, LP3, 13009 Marseille, France
}

(Dated: 30 May 2019)

\begin{abstract}
Spatial segregation of species presents one of the main challenges in quantitative spectroscopy of laser-produced plasmas, as it may lead to overestimation of the concentration of the heavier species. Analytical capabilities can also be affected by excessive Stark broadening at atmospheric pressure, hindering the ability to spectrally resolve closely spaced spectral lines, such as those belonging to isotopes of the same element. We present an experimental and modeling study of the segregation of species and spectral line broadening in the $\mathrm{D}_{2} \mathrm{O}-\mathrm{H}_{2} \mathrm{O}$ plasma produced by single- and double-pulse nanosecond laser ablation in air. The ability to resolve Balmer spectral lines of hydrogen and deuterium is investigated by considering the effects of plume segregation. Transient plasma properties which lead to improvements in spectral line separation are discussed. While the plume segregation is found to be negligible in air regardless of the ablation scheme used, we observe a significant improvement in separation of isotopic spectral lines by employing the double-pulse excitation. This study may lead to increased reliability of optical emission spectroscopy in deuterium-rich plasma environments and suggests the potential for sensitive detection of tritium in air via laser-induced breakdown spectroscopy.
\end{abstract}

Keywords: Deuterium-hydrogen plasma, Balmer alpha, isotope shift, double-pulse LIBS

\section{INTRODUCTION}

The ability to perform standoff detection of radionuclides is relevant in fields such as nuclear security and radiological safety. Tritium is produced in large quantities in nuclear reactors, nuclear tests, and is a vital component of several modern nuclear weapon designs. Moreover, tritium is the fuel of choice for prototype firstgeneration fusion reactors based on either magnetic or inertial confinement fusion. Significant quantities of tritium entering the human body could adversely affect health, increasing the probability of occurrence of cancer, developmental abnormalities, or reproductive defects $^{1}$. Tritium can be detected using low-energy charged particle radiation detectors. However, the feasibility of this method is limited; the mean free path of beta particles produced in tritium decay is on the order of several mm in $\mathrm{air}^{2}$. Therefore, practical detection of tritium poses a challenge not only because of the low rate of characteristic beta particle emission, but also because no highly penetrating, longer-range ionizing radiation, such as gamma rays, is emitted in tritium decay. Laser-induced breakdown techniques may overcome this limitation of detection range and a relatively low signal intensity associated with the traditional radiation detection method. Standoff delivery of a laser interrogation source is commonplace, and bright laser-induced plasmas are sources of abundant characteristic optical signal, making the laserinduced breakdown techniques well-suited for detection

a) Electronic mail: milosb@umich.edu of various radionuclides, including tritium. Here we investigate such an approach based on the optical emission spectroscopy of laser-induced plasma ${ }^{3-5}$, with an emphasis on detection of radioisotopes of interest in mixtures containing multiple isotopes of the same element. This technique, commonly referred to as laser-induced breakdown spectroscopy (LIBS), presents a promising pathway towards remote detection of hazardous radionuclides, especially in conditions where traditional radiation detection fails or performs poorly.

Due to the high cost and difficulty of obtaining tritium, we use deuterium as a proxy. One of the challenges in employing LIBS to identify isotopic species is the degree of separation between closely spaced $(\sim \mathrm{pm})$ spectral lines. The peak-to-peak separation between tritium $\left(\mathrm{T}_{\alpha}\right)$ and hydrogen $\left(\mathrm{H}_{\alpha}\right)$ Balmer alpha lines is $235 \mathrm{pm}$, whereas the peak-to-peak separation between deuterium $\left(\mathrm{D}_{\alpha}\right)$ and hydrogen Balmer alpha lines is $176 \mathrm{pm}$. The ability to resolve these spectral features is made more difficult by excessive Stark and Doppler broadening ${ }^{6,7}$, even when highresolution spectroscopic instrumentation is available. A variation of the LIBS technique, laser-ablation molecular isotopic spectrometry (LAMIS), uses the isotope shift in the optical emission features of molecules, which is typically greater than the atomic isotope shift ${ }^{8,9}$. Despite these difficulties, previous work demonstrates that isotopic discrimination that uses atomic spectral lines is feasible via $\mathrm{LIBS}^{10,11}$. Prior related efforts of several research groups include the quantitative detection of deuterium and hydrogen retained in various fusion reactor components $^{12-15}$.

Detection in ambient air ${ }^{16,17}$ is the most relevant scenario for application of LIBS to detect radionuclides. 
However, detection in air poses several challenges for quantitative analysis, such as strong continuum radiation, plasma nonuniformity ${ }^{18}$, and self-absorption ${ }^{19}$ that limit the ability to distinguish the characteristic isotope spectral features. The smaller volume and larger initial number density for plasmas confined in atmospheric pressure environments appear advantageous, since both parameters contribute to the lower optical thickness ${ }^{20,21}$. In addition, the larger electron density favors the establishment of local thermodynamic equilibrium $(\mathrm{LTE})^{22}$, where collisional processes are more prominent than radiative processes by an order of magnitude. However, as the plasma expands, on the timescale of $\mu \mathrm{s}$ in air ${ }^{4}$, deviations from LTE are expected to arise depending on the electron number density and its transient rate of decay $^{23}$. Although hydrogen, deuterium, and tritium have different atomic masses, their excitation energies are similar, and relative spectral line intensities are nearly independent of the plasma parameters (temperature and electron density). Hydrogen and deuterium are readily observable using optical emission spectroscopy at significant deuterium concentrations under appropriate conditions. However, to detect lower deuterium concentrations using this method, it is necessary to reduce the intense continuum (recombination) emission and excessive Stark broadening, as well as enhance the characteristic line emission signal. Continuum emission and Stark broadening are usually addressed by optimizing the detector gate delay $^{24}$; for standard single-pulse (SP) LIBS, the degree of broadening is typically the lowest at later times, because the plasma is less dense and cooler. The trade-off encountered in this approach is that the line intensity is also reduced, for the same reasons. Under reduced ambient pressure, species segregation in the plasma has also been shown to play a significant role, as the lighter species expand faster into surrounding gas ${ }^{25}$. Since this expansion towards the colder plasma region implies a faster line emission decay, it tends to reduce the spectral line intensities of lighter species relative to heavier ones, and it thus overestimates the relative concentration of heavier plume constituents. The aim of this work is to investigate the effects of species segregation in laser-produced deuteriumhydrogen plasma on the ability to resolve $\mathrm{D}_{\alpha}$ and $\mathrm{H}_{\alpha}$ isotope features and accurately reconstruct their relative concentrations. We further explore the experimental parameters which improve the capability to separate $\mathrm{D}_{\alpha}$ and $\mathrm{H}_{\alpha}$ spectral lines by employing collinear double-pulse (DP) LIBS. DP-LIBS offers additional parameters that can be used to enable a finer control of plasma properties: ablation pulse and reheating pulse configuration, powers and wavelengths of the two pulses, and inter-pulse delay. Signal enhancement employing DP-LIBS has been demonstrated for both nanosecond ${ }^{26}$ and femtosecond laser-produced plasmas ${ }^{27}$. The results of this work are relevant for advancing plasma diagnostics in deuteriumrich environments as well as for applications in standoff detection of deuterium and tritium.

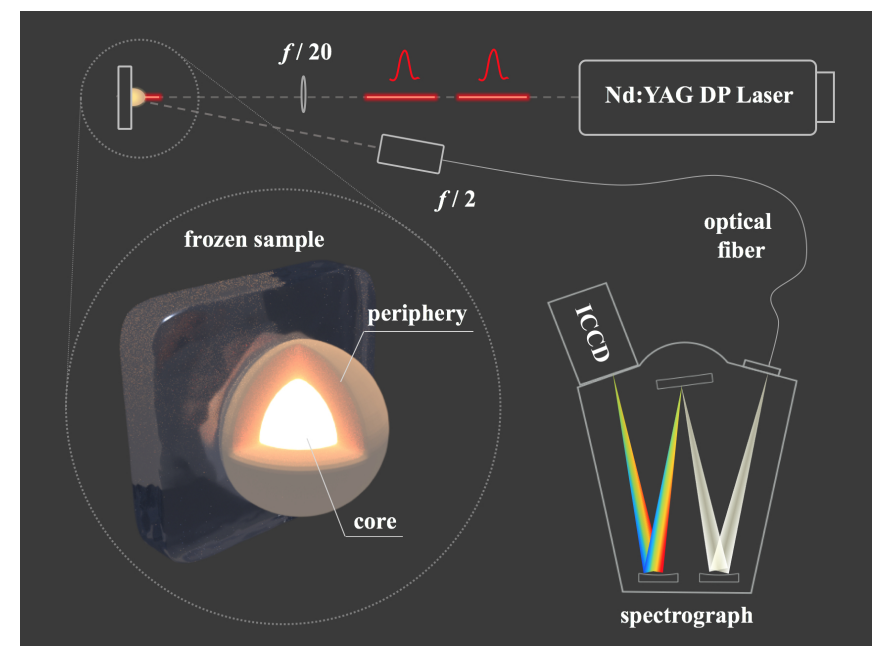

FIG. 1. Schematic of the experimental setup for performing double-pulse LIBS on frozen $\mathrm{D}_{2} \mathrm{O}-\mathrm{H}_{2} \mathrm{O}$ mixture. The magnified section depicts the two-zone approximation used for calculating radiative characteristics of the plasma.

\section{EXPERIMENT}

The experiments (Fig. 1) were performed with a compact, DP Nd:YAG laser (Quantel, model Evergreen 70), producing $\sim 10$-ns, up to $140-\mathrm{mJ}$ single pulses at the wavelength of $1064 \mathrm{~nm}$ and $10-\mathrm{Hz}$ repetition rate. The SP ablation experiments used $75-\mathrm{mJ}$ pulses, whereas DP experiments used $55 \mathrm{~mJ}$ and $75 \mathrm{~mJ}$ for the first and second pulse, respectively. Interpulse (IP) delay in the DP configuration was varied between short (50 ns) and long (1000 ns). The laser beams were focused onto the sample surface using a plano-convex lens with a 100$\mathrm{mm}$ focal length. The laser spot was measured to have a $0.3-\mathrm{mm}$ diameter and a smooth beam profile, corresponding to a laser irradiance of $\sim 10^{9} \mathrm{~W} \mathrm{~cm}^{-2}$ on the sample surface. The samples were prepared by mixing deuterated water $\left(\mathrm{D}_{2} \mathrm{O}\right)$ with deionized light water. The atomic fraction of deuterium in the mixture was set to $40 \%$. The frozen mixture was mounted on a $40 \times 40 \mathrm{~mm}^{2}$ Peltier cooler to ensure the longevity of the $3-\mathrm{mm}$ thick samples. A translation stage was used for continuous sample movement during the measurements. All measurements were performed in air at atmospheric pressure $\left(10^{5} \mathrm{~Pa}\right)$. The plasma emission was collected with an $f / 2$ collimator (Andor, model CC52) and coupled into an optical fiber bundle. The optical axis of the collimator was tilted by $20^{\circ}$ with respect to the laser incidence axis. The fiber bundle was coupled to the 50- $\mathrm{mm}$ slit of a Czerny-Turner spectrometer (Horiba, model iHR550, 1800 lines/mm grating) with a $0.55-\mathrm{m}$ focal length and a resolving power greater than $10^{4}$. The detector was an intensified charge-coupled device matrix detector (Andor, model iStar $334 \mathrm{~T}$ ) cooled down to $-25^{\circ} \mathrm{C}$. Spectral calibration was performed using a $\mathrm{Hg}$-Ar lamp. The gate width was set to $1 \mu \mathrm{s}$, while the gate delay was varied in order to find the measurement timing for an optimized signal acquisition. Spectroscopic data acquisition was performed by averaging over 200 ablation events. Plasma imaging (1:1) was performed by integrating over 30 abla- 


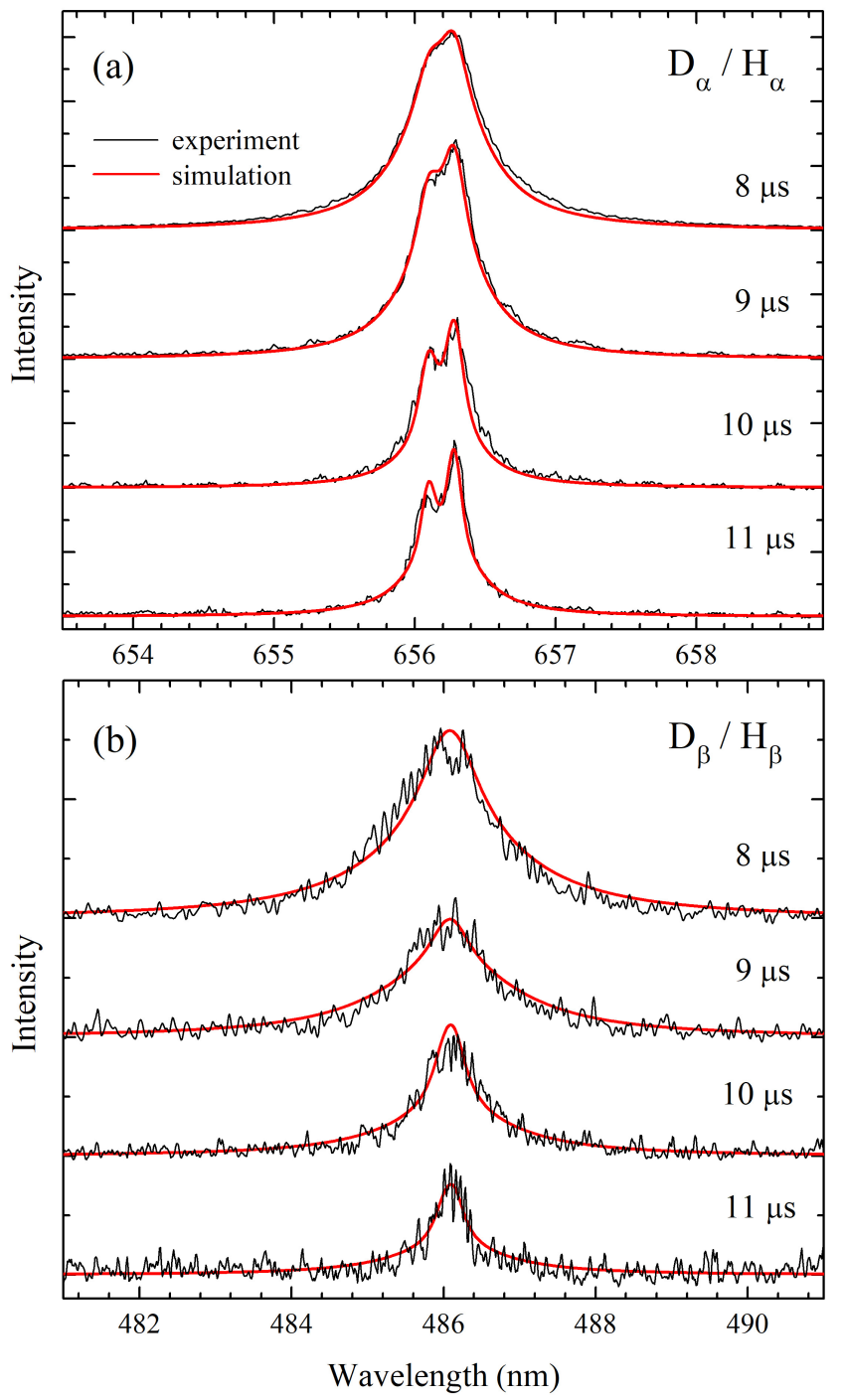

FIG. 2. Measured and computed spectral line profiles of hydrogen-deuterium plasma for Balmer (a) alpha and (b) beta spectral lines at various delays after the laser pulse in the SP configuration.

tion events using a 50-mm diameter, 75-mm focal length achromatic lens and an ICCD detector operating with a gate width of $3 \mathrm{~ns}$.

\section{MODELING DETAILS}

A simplified view of a plasma produced by nanosecond pulses is displayed in Fig. 1, in which the plume is approximated by a hemispherical shape. To consider the spatial variations of temperature and densities, the plasma is divided into two zones: the plasma core and the peripheral zone. Due to the interaction with the background gas (air), the peripheral zone is usually colder than the plasma core. Compared to the approximation of uniform plasma, the introduction of peripheral zone presents a first-order correction in calculations of the plasma emission spectrum. It is assumed that the cold peripheral region absorbs some of the emission from the core. The spectral radiance can be computed using an analytical solution of the radiation transfer equation. If the spectral radiance is observed in the $z$ (laser) direction, and a plasma in local thermodynamic equilibrium is assumed, we have ${ }^{28}$

$$
I(\lambda)=U_{p}\left(1-e^{-\tau_{p}}\right)+U_{c}\left(1-e^{-\tau_{c}}\right) e^{-\tau_{p}},
$$

where $U$ is the blackbody spectral radiance and $\tau$ is the optical thickness that characterizes the transmissive properties of the plasma. The subscripts $c$ and $p$ represent the core and peripheral plasma zones, respectively. The optical thickness in direction $z$ is given by

$$
\tau=\int_{0}^{L} \alpha(z) d z=\alpha L
$$

where $L$ is diameter of the plasma zone along the observation direction and $\alpha$ is the absorption coefficient given by $^{6}$

$$
\alpha(\lambda)=\pi r_{0} \lambda^{2} f_{l u} n_{l} P(\lambda)\left(1-e^{-h c / \lambda k T}\right) .
$$

Here, $r_{0}$ is the classical electron radius, $h$ is the Planck constant, $c$ is the vacuum light velocity. The lower-level population number density $\left(n_{l}\right)$ of the transition is deduced from the LTE-based calculations ${ }^{29}$. The absorption oscillator strength $\left(f_{l u}\right)$ relates to Einstein coefficient for spontaneous emission $\left(A_{l u}\right)$ as $^{30}$

$$
f_{l u}=\frac{\varepsilon_{0}}{e^{2}} \frac{m_{e} c^{3}}{2 \pi \nu_{l u}^{2}} \frac{g_{l}}{g_{u}} A_{l u},
$$

where $\varepsilon_{0}$ is the vacuum permittivity, $\nu_{l u}$ is the frequency of transition, $g_{l}$ and $g_{u}$ are the statistical weights of lower and upper levels (respectively), and $m_{e}$ and $e$ are the electron mass and charge, respectively. The normalized line profile $P(\lambda)$ is calculated by including the Doppler and Stark effects, the dominant mechanisms of spectral line broadening in strongly ionized laser-produced plasmas ${ }^{31}$. Depending on the relative Doppler and Stark widths, the line shapes are described by Gaussian, Lorentzian, or Voigt profiles ${ }^{7}$. The Doppler width is calculated according to plasma temperature and atomic mass of the emitting species. The Stark width of each spectral line can be obtained using the previously determined Stark broadening parameter $w$ while assuming a following dependence of Stark width with the electron density ${ }^{32}$ :

$$
\Delta \lambda_{S}=w\left(\frac{n_{e}}{n_{e}^{r e f}}\right)^{m}
$$

where $n_{e}^{r e f}$ is the reference electron density for given $w$. A linear dependence of Stark width on electron density $(\mathrm{m}=1)$ can be assumed for non-hydrogenic lines. In the present work, we use the expression proposed by Gigosos et al. ${ }^{33,34}$. We deduce electron density and temperature for both the plasma core and the border by comparing experimental with simulated profiles. The atomic fraction of deuterium was set to $40 \%$ in the simulation. The atomic structure of hydrogen is used for both hydrogen and deuterium. We therefore compute two profiles 


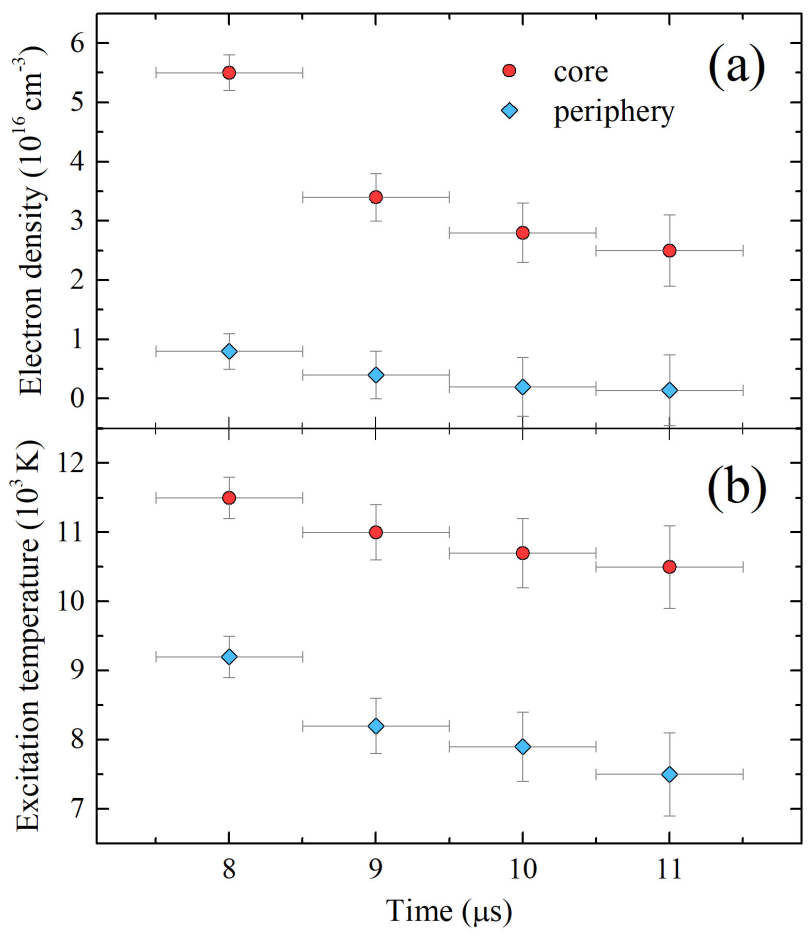

FIG. 3. Temporal evolution of (a) electron density and (b) excitation temperature of the plasma core and periphery in the $\mathrm{SP}$ configuration

at their corresponding positions; the population number densities of upper and lower levels are shared according to the relative isotope fractions. The line center positions for $\mathrm{H}_{\alpha}, \mathrm{D}_{\alpha}$ and $\mathrm{H}_{\beta}$ were taken from the published data ${ }^{35}$, whereas the $\mathrm{D}_{\beta}$ component positions were calculated by using an expression for wavelength $(\lambda)$ associated with transition from upper $\left(n_{u}=4\right)$ to lower $\left(n_{l}=2\right)$ level:

$$
\frac{1}{\lambda_{D}}=R\left(\frac{1}{n_{l}^{2}}-\frac{1}{n_{u}^{2}}\right)\left(1-\frac{m_{e}}{M}\right),
$$

where $R$ is the Rydberg constant, $c$ is the speed of light, $m_{e}$ is the electron mass, and $M$ is the isotope mass.

\section{RESULTS AND DISCUSSION}

\section{A. Single pulse}

Laser-produced plasmas formed in air often exhibit a high degree of spatial nonuniformity, leading to more pronounced self-absorption in the cooler peripheral plasma regions. Self-absorption becomes more prominent at later observation times, thereby seriously distorting the line emission profiles and the accuracy of plasma diagnostics at these delays. We focus our analysis on the time period in which the isotopic splitting of hydrogen lines can be observed. For more precise measurements of electron density, the line shape of the Balmer beta transition was analyzed. This line has been well investigated ${ }^{34,36,37}$ and is known to provide reliable electron density. Furthermore, it is less prone to self-absorption compared to the more intense Balmer alpha line. At later delay times, plasma deviates from LTE, especially at the plasma periphery. In order to account for plasma nonuniformity, we describe the plasma by dividing it into two zones of different thermodynamic states, representing the hot core and cold periphery ${ }^{19}$, as discussed in the previous section. Fig. 2 shows the SP experimental and computed profiles of superimposed hydrogen and deuterium lines from the onset of spectral splitting ( $8 \mu \mathrm{s})$ to the final stage of the Balmer beta lifetime (11 ps). The good agreement between experimental and simulated profiles indicates a negligible change in isotopic ratio at this time interval. The electron density and excitation temperature trends were deduced from the simulated profiles, including contributions of plasma core and peripheral region (Fig. 3). Typical electron densities and excitation temperatures are on the order of $10^{16} \mathrm{~cm}^{-3}$ and $10^{4} \mathrm{~K}$, respectively. We note that the difference between the electron density of the core and the periphery decreases with time, whereas the excitation temperature difference exhibits the opposite trend. This can be explained by increased diffusion and gradual deviation from equilibrium state, which are both characteristic for the late observation times. Behavior of isotopic ratio at even later observation times is addressed in the following subsection for SP and DP configurations.

\section{B. Double pulse}

We employ DP ablation in order to increase signalto-noise ratio and improve the ability to distinguish the hydrogen isotopic spectral lines. Ideally, one would want to simultaneously reduce Stark broadening (electron density) and increase the plasma temperature in order to better resolve $\mathrm{D}_{\alpha}$ from $\mathrm{H}_{\alpha}$. We employ a collinear DP scheme, in which we set the energy of the first pulse to be significantly lower than the second pulse. This scheme allows the plasma to form with lower initial density at atmospheric pressure $\left(10^{5} \mathrm{~Pa}\right)$. The majority of the energy of the second, reheating pulse, is absorbed by the vapor plume created by the first pulse. This process is especially efficient at infrared wavelengths, since the inverse bremsstrahlung rate scales quadratically with laser wavelength, thereby reducing the amount of energy reaching the target surface ${ }^{38}$. Two IP delays were used in order to investigate the effects of laser-plasma coupling on line broadening. Compared to the long (1000-ns) IP delay, the short (50-ns) IP delay takes advantage of the rarefied conditions within the shockwave boundary during the early times of plasma formation and expansion. It has been shown in a previous study ${ }^{39}$, that this approach may reduce Stark broadening. Fig. 4 shows time-resolved $\mathrm{D}_{\alpha}$ and $\mathrm{H}_{\alpha}$ emission isolated via narrow-band interference filter centered at $656 \mathrm{~nm}$ (10-nm bandwidth) for SP and DP ablation schemes. Fig. 4 also compares the short and long IP delays for the DP scheme. The arrival of the second laser pulse in the short IP case causes elongation of the plume along the laser axis, whereas the second pulse in the long IP case causes a short-lived feature at the front edge of the plume, while having a negligible ef- 


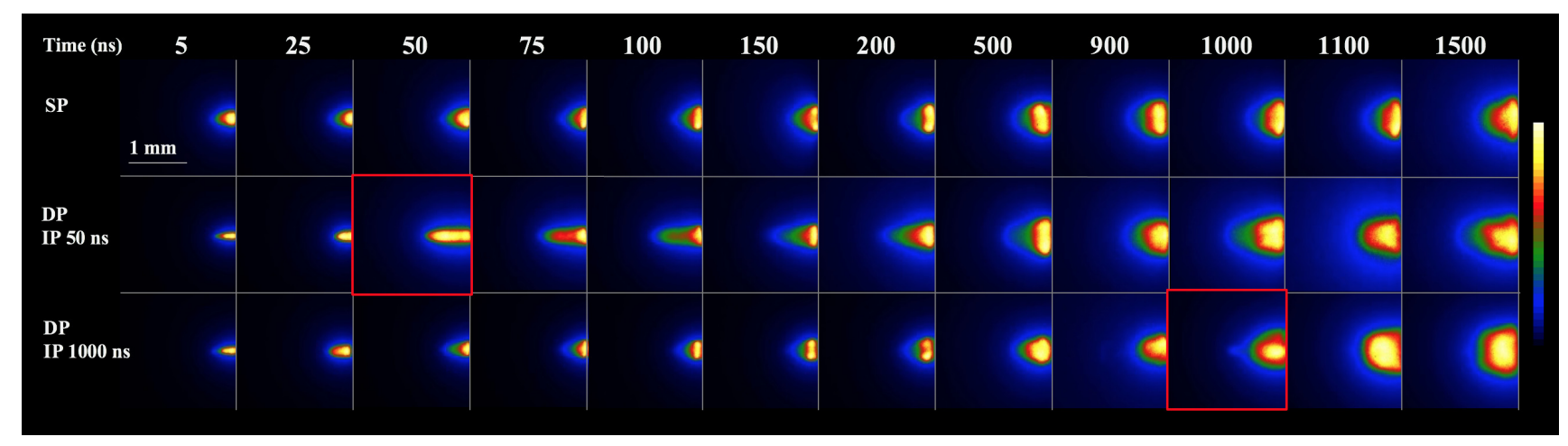

FIG. 4. Time-resolved, filtered plasma emission comparing SP and DP ablation with short (50-ns) and long (1000-ns) IP delays. The spectral interference filter is centered at $656 \mathrm{~nm}$ with $10-\mathrm{nm}$ bandwidth and it isolates the $\mathrm{D}_{\alpha}$ and $\mathrm{H}_{\alpha}$ emission. Each image shows an accumulation of 30 shots. The gate width used is 3 ns. The red frame outline indicates the arrival of the second pulse.

fect on the plume shape at later times. The effects of the second pulse on the plume shape for the short IP delay imply some secondary ablation as well as a significant increase in temperature. The negligible change of the plume shape seen in the long IP case indicates better laser-plasma coupling and a reduced rate of secondary ablation.

Fig. 5 compares late-time emission between the SP and DP ablation schemes. In the case of short IP delay, the persistence of observed lines increases to $\sim 22 \mu \mathrm{s}$ from $\sim 15 \mu$ in the SP case, despite the lower energy of the initial pulse. The increased persistence is attributed to the greater plasma temperature upon reheating with the second pulse. In the long IP case, the persistence time is $\sim 14 \mu \mathrm{s}$, shorter than in the SP case. The second laser pulse in this scheme appears to have less effect on the plasma temperature upon the arrival of the second pulse. This may imply better laser-plasma coupling after the initial plasma has become larger, such that a greater fraction of the incoming laser energy is absorbed and the secondary ablation rate is reduced.

Mercadier et al..$^{25}$ observed notable segregation in lowpressure environments $\left(10^{2} \mathrm{~Pa}\right)$ and predicted a smaller contribution at greater pressures. We investigate the contribution of segregation at atmospheric pressure by observing the time variation in the relative intensities of the deuterium and hydrogen emission features. Fig. 6(a) compares the $\mathrm{D}_{\alpha}$ line intensity to the total intensity of both $\mathrm{D}_{\alpha}$ and $\mathrm{H}_{\alpha}$ at a fixed observation position. The ratio remains nearly constant regardless of the configuration used, implying negligible plume segregation for ambient pressure $10^{5} \mathrm{~Pa}$. This result confirms the hypothesis from Ref. ${ }^{25}$, predicting a minor role of plume segregation under atmospheric pressure. The axial plume front position deduced from the plume images (Fig. 4) is shown in Fig. 7 as a function of time for the three excitation schemes. Stagnation of the neutral hydrogen and deuterium species is implied by deceleration of the plume front toward later times, supporting the conclusion that the species segregation is negligible.

Fig. 6 (a) shows one of the possible figures of merit (FOM) for distinguishing deuterium from hydrogen; the value of this FOM at selected delays is provided in Ta- ble I (a). We note the relatively constant trend for all ablation schemes, signifying the absence of notable segregation effects. Finally, we assess the ability to distinguish the isotopes by measuring the line broadening of both $\mathrm{D}_{\alpha}$ and $\mathrm{H}_{\alpha}$ lines for each ablation scheme. The FOM in Fig. 6 (b) characterizes the degree of broadening normalized to the peak-to-peak separation $d$, which limits the ability to resolve the $\mathrm{D}_{\alpha}$ from $\mathrm{H}_{\alpha}$ Balmer lines:

$$
\mathrm{FOM}=\frac{d}{w_{D}+w_{H}},
$$

where $w_{D}$ and $w_{H}$ represent the measured half-widths for $\mathrm{D}_{\alpha}$ and $\mathrm{H}_{\alpha}$ lines, respectively. The measurement error of isotopic ratio depends on the FOM, but also on the signal-to-noise ratio. It is difficult to infer the plasma density and temperature at late delays by measuring line broadening due to prominent self-absorption in the high-pressure environment. Nonetheless, we assume equal probability for self-absorption for both $\mathrm{D}_{\alpha}$ and $\mathrm{H}_{\alpha}$ lines and quantify the net effect parametrized by the FOM. We observe the greatest FOM (which implies better distinction between $\mathrm{D}_{\alpha}$ and $\mathrm{H}_{\alpha}$ lines) for long IP delay at earlier time steps. However, the second pulse does not sufficiently reheat the existing plasma to enhance the persistence beyond that of the SP or short IP cases. The SP broadening quickly diminishes and ultimately the short IP delay exhibits the greatest FOM after 20 us.

\section{v. CONCLUSION}

We studied the effects of species segregation and spatial nonuniformity in laser-produced plasmas formed in air in order to distinguish between deuterium and hydrogen Balmer alpha lines. We simulated the conditions in the plasma for the SP configuration in order to determine the plasma properties which govern the emission from nonuniform plasma by using a two-temperature zone model. The agreement between experimental data and simulation implies that the isotopic fraction remains unchanged during the Balmer beta spectral line lifetime. Ultimately, we observe considerable broadening in the 


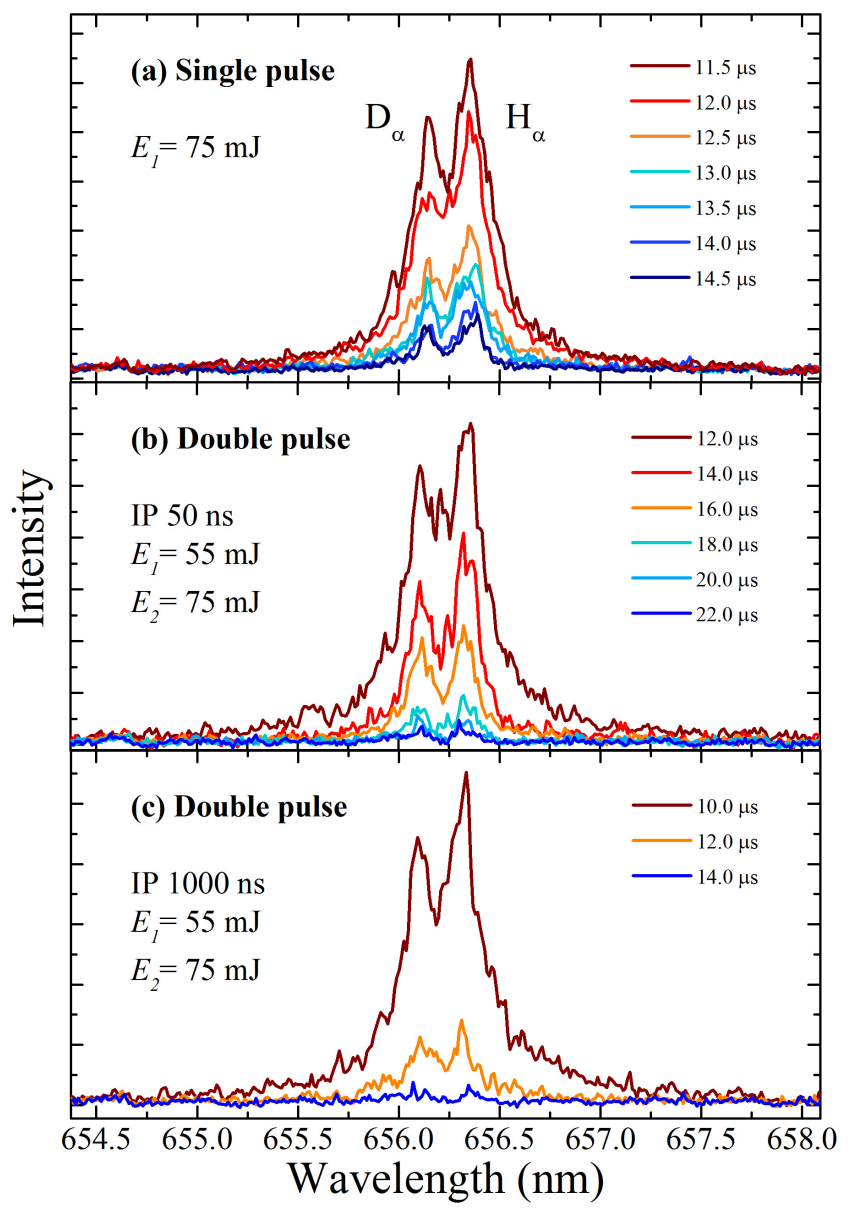

FIG. 5. Late-time emission of $\mathrm{D}_{\alpha}(656.103 \mathrm{~nm})$ and $\mathrm{H}_{\alpha}$ $(656.279 \mathrm{~nm})$ for (a) SP and DP using (b) short (50-ns) and (c) long (1000-ns) IP delays. The SP energy $E_{1}$ was $75 \mathrm{~mJ}$, whereas energies for the first $\left(E_{1}\right)$ and second $\left(E_{2}\right)$ pulse were $55 \mathrm{~mJ}$ and $75 \mathrm{~mJ}$, respectively. Spectra were accumulated over 200 laser shots.

SP configuration, which limits the ability to distinguish $\mathrm{D}_{\alpha}$ and $\mathrm{H}_{\alpha}$ lines to a narrow temporal window ( $\left.\sim \mu \mathrm{s}\right)$ at late delays, up to $\sim 14 \mu \mathrm{s}$. We attempted to reduce the degree of Stark broadening and simultaneously enhance the line intensity by employing a DP configuration. We investigated two IP delays, short (50 ns) and long (1000 ns), in order to determine how laser-plasma coupling affects the Stark broadening and plasma uniformity. We find that species segregation for laser-produced plasma formed in air does not significantly impact the ability to distinguish $\mathrm{D}_{\alpha}$ and $\mathrm{H}_{\alpha}$ Balmer lines for both the SP and DP laser ablation schemes. Rapid deceleration of the plume front for each ablation scheme suggests the atmospheric-pressure environment leads to a larger degree of plasma confinement and species stagnation in contrast to plasmas formed at low backing pressures, as discussed by Mercadier et al. ${ }^{25}$. Significant reduction of broadening was observed in comparison to the SP configuration by employing the second, reheating pulse in a collinear geometry while using a lower-energy initial pulse. We describe the net degree of broadening by introducing the FOM parameter, which compares the ob-
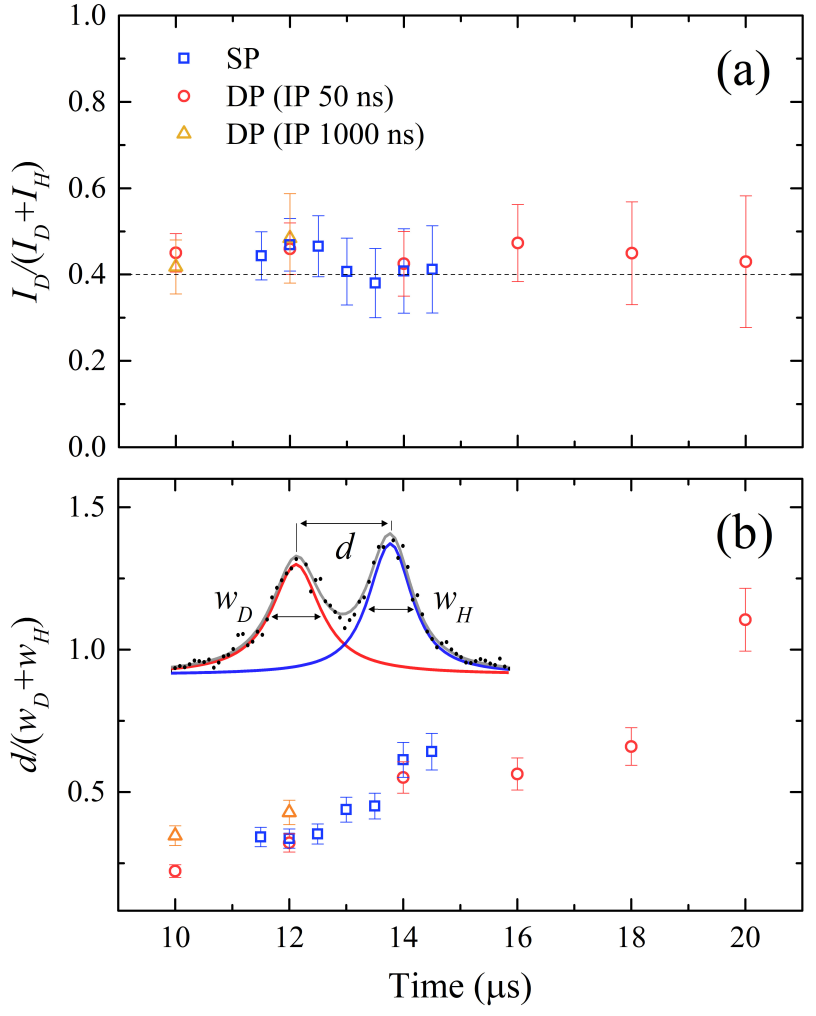

FIG. 6. Figure of merit for distinguishing deuterium from hydrogen: (a) based upon the relative intensities of the $\mathrm{D}_{\alpha}$ line $\left(I_{D}\right)$ and the $\mathrm{H}_{\alpha}$ lines $\left(I_{H}\right)$; (b) based upon line widths of the $\mathrm{D}_{\alpha}$ line $\left(w_{D}\right)$ and the $\mathrm{H}_{\alpha}$ line $\left(w_{H}\right)$ and their isotopic separation $d$. The dashed line indicates the intensity ratio expected for the isotopic ratio of the sample.

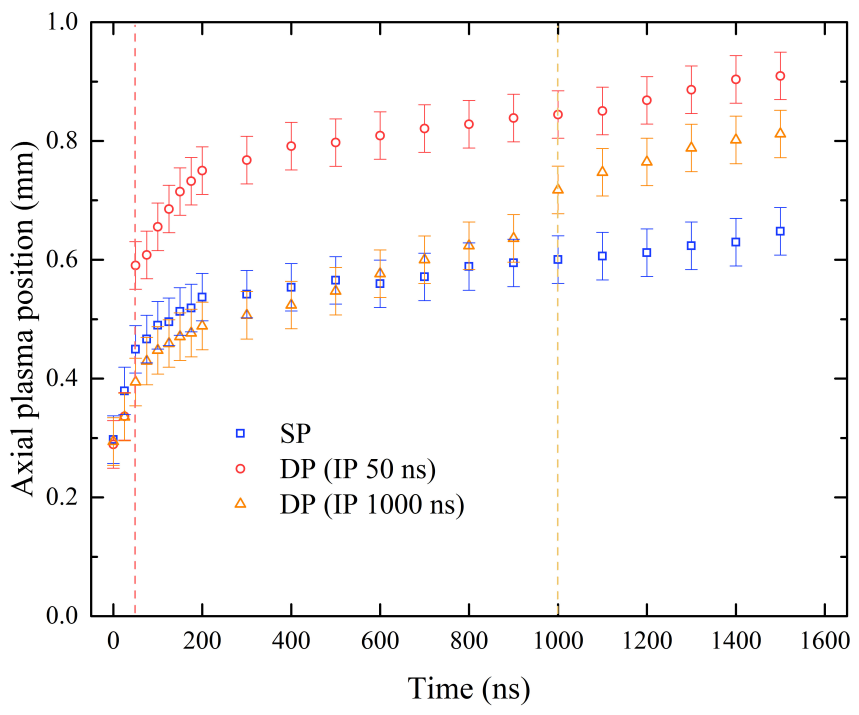

FIG. 7. Axial position of the plasma plume front as a function of time for each ablation scheme. Only the total deuterium $\left(\mathrm{D}_{\alpha}\right)$ and hydrogen $\left(\mathrm{H}_{\alpha}\right)$ emission is tracked. Vertical dashed lines mark the arrival of the second laser pulse for short (50ns) and long (1000-ns) IP delays. 
TABLE I. Measured isotopic ratio $I_{D} /\left(I_{d}+I_{H}\right)$ at selected delays

\begin{tabular}{lccc}
\hline \hline Time $(\mu \mathrm{s})$ & SP & DP (IP 50 ns) & DP (IP 1000 ns) \\
\hline 10 & - & $0.45 \pm 0.045$ & $0.42 \pm 0.063$ \\
12 & $0.46 \pm 0.06$ & $0.46 \pm 0.060$ & $0.48 \pm 0.10$ \\
14 & $0.41 \pm 0.10$ & $0.43 \pm 0.075$ & - \\
16 & - & $0.47 \pm 0.089$ & - \\
18 & - & $0.45 \pm 0.12$ & - \\
20 & - & $0.43 \pm 0.15$ & - \\
\hline \hline
\end{tabular}

served line widths to the peak-to-peak line separation. The long IP delay exhibits a greater FOM, implying a smaller net broadening and better ability to resolve the two spectral features, than both the SP and the shortIP-delay DP ablation scheme; however, the second pulse does not sufficiently extend the persistence of the Balmer alpha transitions for either species beyond even that of the SP case. On the other hand, the short-IP-delay DP excitation significantly improves both the persistence as well as demonstrates the best distinction between $\mathrm{D}_{\alpha}$ and $\mathrm{H}_{\alpha}$ lines at late delay times. The practical aspect of collinear DP geometry is favorable for in-situ applications. Therefore, DP-LIBS presents a promising pathway towards standoff detection of hazardous low-Z radionuclides, such as tritium, in air. Further studies involving optimization of interpulse delay and energy-ratio in DP configuration would be highly beneficial towards potential improvements in both FOM and spectral line persistence, ultimately leading to lower limits of detection.

\section{ACKNOWLEDGMENTS}

We thank K. Kearfott of the University of Michigan for providing the initial motivation for this study. This work was supported by the Consortium for Verification Technology under U.S. Department of Energy National Nuclear Security Administration award number DE-NA0002534.

\section{REFERENCES}

${ }^{1}$ T. Straume and A. Carsten, Health Physics 65, 657 (1993).

${ }^{2}$ J. R. Lamarsh and A. J. Baratta, Introduction to Nuclear Engineering (Prentice Hall, New Jersey, 2001).

${ }^{3}$ B. Kearton and Y. Mattley, Nature Photonics 2, 537 (2008).

${ }^{4}$ R. Noll, Laser-Induced Breakdown Spectroscopy Fundamentals and Applications (Springer-Verlag, Berlin Heidelberg, 2012).

${ }^{5}$ J. Hermann, D. Grojo, E. Axente, C. Gerhard, M. Burger, and V. Craciun, Phys. Rev. E 96, 053210 (2017).

${ }^{6}$ H. R. Griem, Plasma spectroscopy (Academic Press, New York, 1964).

${ }^{7}$ H. R. Griem, Spectral line broadening of plasmas (Academic Press, New York, 1974).

${ }^{8}$ R. E. Russo, A. A. Bol'shakov, X. Mao, C. P. McKay, D. L. Perry, and O. Sorkhabi, Spectrochimica Acta Part B: Atomic Spectroscopy 66, 99 (2011).

${ }^{9}$ G. Herzberg, Molecular Spectra and Molecular Structure: Spectra of Diatomic Molecules, 2nd ed. (Van Nostrand Reinhold, London, 1950).
${ }^{10}$ F. R. Doucet, G. Lithgow, R. Kosierb, P. Bouchard, and M. Sabsabi, J. Anal. At. Spectrom. 26, 536 (2011).

${ }^{11} \mathrm{P}$. Ko and I. Jovanovic, Spectrochimica Acta Part B: Atomic Spectroscopy 90, 68 (2013).

${ }^{12}$ L. Mercadier, J. Hermann, C. Grisolia, and A. Semerok, Journal of Nuclear Materials 415, S1187 (2011).

${ }^{13}$ L. Mercadier, C. Grisolia, H. Roche, A. Semerok, J. Hermann, C. Pocheau, P.-Y. Thro, J.-B. Sirven, J.-M. Weulersse, P. Mauchien, and M. Sentis, Fusion Science and Technology 60, 1049 (2011).

${ }^{14}$ P. Paris, J. Butikova, M. Laan, M. Aints, A. Hakola, K. Piip, I. Tufail, and P. Veis, Physica Scripta 2017, 014003 (2017).

${ }^{15}$ F. Colao, S. Almaviva, L. Caneve, G. Maddaluno, T. Fornal, P. Gasior, M. Kubkowska, and M. Rosinski, Nuclear Materials and Energy 12, 133 (2017).

${ }^{16}$ S. S. Harilal, B. E. Brumfield, and M. C. Phillips, Physics of Plasmas 22, 063301 (2015).

${ }^{17}$ S. S. Harilal, P. J. Skrodzki, A. Miloshevsky, B. E. Brumfield, M. C. Phillips, and G. Miloshevsky, Physics of Plasmas 24, 063304 (2017).

${ }^{18}$ J. Aguilera and C. Aragón, Spectrochimica Acta Part B: Atomic Spectroscopy 59, 1861 (2004).

${ }^{19}$ J. Hermann, A. Lorusso, A. Perrone, F. Strafella, C. Dutouquet, and B. Torralba, Phys. Rev. E 92, 053103 (2015).

${ }^{20}$ D. Bulajic, M. Corsi, G. Cristoforetti, S. Legnaioli, V. Palleschi, A. Salvetti, and E. Tognoni, Spectrochimica Acta Part B: Atomic Spectroscopy 57, 339 (2002).

${ }^{21}$ M. Burger, M. Skočić, and S. Bukvić, Spectrochimica Acta Part B: Atomic Spectroscopy 101, 51 (2014).

${ }^{22}$ S. Eliezer, A. D. Krumbein, and D. Salzmann, Journal of Physics D: Applied Physics 11, 1693 (1978).

${ }^{23}$ G. Cristoforetti, A. D. Giacomo, M. Dell'Aglio, S. Legnaioli, E. Tognoni, V. Palleschi, and N. Omenetto, Spectrochimica Acta Part B: Atomic Spectroscopy 65, 86 (2010).

${ }^{24}$ A. D'Ulivo, M. Onor, E. Pitzalis, R. Spiniello, L. Lampugnani, G. Cristoforetti, S. Legnaioli, V. Palleschi, A. Salvetti, and E. Tognoni, Spectrochimica Acta Part B: Atomic Spectroscopy 61, 797 (2006).

${ }^{25}$ L. Mercadier, J. Hermann, C. Grisolia, and A. Semerok, Spectrochimica Acta Part B: Atomic Spectroscopy 65, 715 (2010).

${ }^{26}$ A. D. Giacomo, M. Dell'Aglio, D. Bruno, R. Gaudiuso, and O. D. Pascale, Spectrochimica Acta Part B: Atomic Spectroscopy 63, 805 (2008).

${ }^{27}$ A. Chen, S. Li, S. Li, Y. Jiang, J. Shao, T. Wang, X. Huang, M. Jin, and D. Ding, Physics of Plasmas 20, 103110 (2013).

${ }^{28} \mathrm{~J}$. Hermann, C. Boulmer-Leborgne, and D. Hong, Journal of Applied Physics 83, 691 (1998).

${ }^{29}$ J. Hermann and C. Dutouquet, J. Appl. Phys. 91, 10188 (2002).

${ }^{30}$ I. Hutchinson, Principles of Plasma Diagnostics, 2nd ed. (Cambridge University Press, 2002).

${ }^{31}$ X. Z. Zhao, L. J. Shen, T. X. Lu, and K. Niemax, Applied Physics B 55, 327 (1992).

${ }^{32}$ E. Tognoni, V. Palleschi, M. Corsi, G. Cristoforetti, N. Omenetto, I. Gornushkin, B. W. Smith, and J. D. Winefordner, Laser-induced Breakdown Spectroscopy (edited by A. W. Miziolek, V. Palleschi, and I. Schechter (Cambridge University Press, Berlin), 2006).

${ }^{33}$ M. A. Gigosos, M. A. Gonzalez, and V. Cardenoso, Spectrochimica Acta Part B: Atomic Spectroscopy 58, 1489 (2003).

${ }^{34}$ N. Konjević, M. Ivković, and N. Sakan, Spectrochimica Acta Part B: Atomic Spectroscopy 76, 16 (2012).

${ }^{35}$ A. Kramida, Y. Ralchenko, and J. Reader, "NIST Atomic Spectra Database," (2018), National Institute of Standards and Technology, Gaithersburg, MD.

${ }^{36}$ H. R. Griem, J. Halenka, and W. Olchawa, Journal of Physics B: Atomic, Molecular and Optical Physics 38, 975 (2005).

${ }^{37}$ M. Ivković, N. Konjević, and Z. Pavlović, Journal of Quantitative Spectroscopy and Radiative Transfer 154, 1 (2015).

${ }^{38}$ M. Burger, D. Pantić, Z. Nikolić, and S. Djeniže, Journal of Quantitative Spectroscopy and Radiative Transfer 170, 19 (2016).

${ }^{39}$ S. Beldjilali, W. L. Yip, J. Hermann, T. Baba-Hamed, and A. Belasri, Analytical and Bioanalytical Chemistry 400, 2173 (2011). 